\title{
Electronic structure of pristine and K-doped solid picene: Nonrigid band change and its implication for electron-intramolecular-vibration interaction
}

\author{
H. Okazaki, ${ }^{1,2}$ T. Wakita, ${ }^{1,2}$ T. Muro, ${ }^{3}$ Y. Kaji, ${ }^{1}$ X. Lee, ${ }^{1}$ H. Mitamura, ${ }^{1}$ N. Kawasaki, ${ }^{1}$ Y. Kubozono, ${ }^{1}$ Y. Yamanari, ${ }^{1}$ \\ T. Kambe, ${ }^{1}$ T. Kato, ${ }^{4}$ M. Hirai, ${ }^{1}$ Y. Muraoka, ${ }^{1,2}$ and T. Yokoya ${ }^{1,2}$ \\ ${ }^{1}$ The Graduate School of Natural Science and Technology, Okayama University, 3-1-1 Tsushima-naka, Okayama 700-8530, Japan \\ ${ }^{2}$ CREST, Japan Science and Technology Corporation (JST), 3-1-1 Tsushima-naka, Okayama 700-8530, Japan \\ ${ }^{3}$ Japan Synchrotron Radiation Research Institute (JASRI)/SPring-8, 1-1-1 Kouto, Sayo, Hyogo 679-5198, Japan \\ ${ }^{4}$ Institute for Innovative Science and Technology, Graduate School of Engineering, Nagasaki Institute of Applied Science, \\ 3-1, Shuku-machi, Nagasaki 851-0121, Japan
}

(Received 27 August 2010; revised manuscript received 14 October 2010; published 10 November 2010)

\begin{abstract}
We use photoemission spectroscopy to study electronic structures of pristine and K-doped solid picene. The valence band spectrum of pristine picene consists of three main features with no state at the Fermi level $\left(E_{\mathrm{F}}\right)$ while that of K-doped picene has three structures similar to those of pristine picene with new states near $E_{\mathrm{F}}$, consistent with the semiconductor-metal transition. The K-induced change cannot be explained with a simple rigid-band model of pristine picene but can be interpreted by molecular-orbital calculations considering electron-intramolecular-vibration interaction. Excellent agreement of the K-doped spectrum with the calculations points to importance of electron-intramolecular-vibration interaction in K-doped picene.
\end{abstract}

DOI: 10.1103/PhysRevB.82.195114

PACS number(s): 79.60.-i, 74.25.Jb, 72.80.Le, 63.20.kd

\section{INTRODUCTION}

Carbon systems have attracted much attention because of the discovery of new superconductors with relatively high superconducting transition temperatures; heavily borondoped diamond with $T_{\mathrm{c}}<10 \mathrm{~K}, 1,2$ graphite intercalation compound superconductor $\mathrm{CaC}_{6}$ with $T_{\mathrm{c}}=11.5 \mathrm{~K},{ }^{3}$ and alkali-doped fullerene $\mathrm{Cs}_{3} \mathrm{C}_{60}$ with $T_{\mathrm{c}}=38 \mathrm{~K}$ under $7 \mathrm{GPa}^{4}$ Recently, a new type of superconductors was discovered in a solid of aromatic molecule picene, with $T_{\mathrm{c}}=7$ and $18 \mathrm{~K}$ when doped with potassium $(\mathrm{K}){ }^{5}$ The discovery of superconducting aromatic molecule picene motivates researchers to search for new aromatic molecular superconductors with higher $T_{\mathrm{c}}$ as well as to understand the mechanism of the superconductivity. A picene molecule consists of five benzene rings sharing some of their edges, forming a zigzag structure, and solid picene has a layer structure stack to $c$ axis, where each layer has a herringbone structure with the picene molecules inclining a little from the $a b$ plane. ${ }^{6}$ Thus, the structure is two dimensional. Solid picene is remarkable for its physical properties; wide band gap of $3.3 \mathrm{eV}$ (Ref. 7) and high carrier mobility of greater than $3 \mathrm{~cm}^{2} \mathrm{~V}^{-1} \mathrm{~s}^{-1}$ by exposure to $\mathrm{O}_{2}{ }^{8}$ However, neither the electronic structure of the pristine nor doped picene has been reported, yet. It is, therefore, crucial to study experimental electronic structure of pristine picene and its evolution with doping in order to understand the mechanism of superconductivity.

For alkali-doped superconducting fullerene, which is similar to molecular crystal as picene, it is considered that the electrons of doped alkali atoms are transferred to the threefold degenerated lowest unoccupied molecular orbital (LUMO) bands of fullerene. However, the electrons do not rigidly occupy the LUMO bands of fullerene, instead the LUMO bands split into the occupied and unoccupied states ${ }^{9}$ by Jahn-Teller distortion, ${ }^{10}$ which originates from a coupling between electrons doped into the LUMO and intramolecular phonon of a fullerene molecule. The electron-intramolecular- vibration coupling is considered to be essential in understanding the physical properties of fullerides including superconductivity. ${ }^{10}$ Therefore, the role of electronintramolecular-vibration interaction in K-doped picene should be investigated in order to understand the superconductivity.

In this paper, we report photoemission spectroscopy (PES) of pristine and K-doped picene films in order to elucidate the evolution of electronic structure by $\mathrm{K}$ doping. A valence band spectrum of K-doped picene shows new states near $E_{\mathrm{F}}$ besides several structures that can be related to those of pristine picene. The states near $E_{\mathrm{F}}$ in K-doped picene can be ascribed to states derived from the LUMO of pristine picene but is not interpreted by a simple rigid shift of the LUMO of pristine picene. The valence band spectrum of $\mathrm{K}$-doped picene is in excellent agreement with the molecular-orbital calculation of negatively charged picene including electron-intramolecular-vibration interactions. This result indicates importance of electron-intramolecularvibration interactions in K-doped picene, which can play a crucial role for the metallic properties leading to superconductivity.

\section{EXPERIMENTAL}

Picene was prepared by photosensitization of 1,2-di(1naphthyl)ethane with 9-fluorenone in chloroform. The details of the procedure are described in a literature. ${ }^{7}$ Pristine picene thin films for the measurements at Hiroshima Synchrotron Radiation Center (HiSOR) were prepared by in situ deposition of picene powder on Au-coated stainless substrates under ultrahigh vacuum. X-ray diffraction of the solid pristine picene films showed that they have the same crystal structure as that of solid picene. $\mathrm{K}$ doping was achieved by successive deposition of potassium onto the pristine picene films for 40 min. The $\mathrm{K}$ concentration $x$ of K-doped picene $\left(\mathrm{K}_{x} \mathrm{C}_{22} \mathrm{H}_{14}\right)$ films in the region measured with present PES was estimated 
to be $1.0 \pm 0.3$ from the spectral weight ratio between $\mathrm{K} 3 p$ and $\mathrm{C} 2 s-p$ derived valence band measured with $100 \mathrm{eV}$, taking the ionization cross sections of $\mathrm{K} 3 p$ and $\mathrm{C} 2 p$ or $\mathrm{C} 2 s$ into consideration ${ }^{11}$ (see later for the detail). For the measurements at SPring-8, ex situ pristine picene films prepared with the same procedure as described above were used. Absence of oxygen-related signals in the pristine picene films ensured no oxygen-related adsorption.

We performed the PES measurements at two different sites in order to distinguish C $2 s$ and $2 p$ orbitals utilizing the difference of cross-section ratio. The PES measurement at BL-5 (Okayama University Beamline) of HiSOR was performed using photon energy of $100 \mathrm{eV}$. We used the $100 \mathrm{eV}$ photon energy in order to obtain high signal to noise ratio, as the intensity of $100 \mathrm{eV}$ photon energy is the highest in this beamline. Another PES measurement at BL27SU of SPring-8 was done using photon energy of $1100 \mathrm{eV}$. The energy resolutions of PES at HiSOR and SPring-8 were set to be $250 \mathrm{meV}$ and $300 \mathrm{meV}$, respectively. All the measurements were performed at room temperature and under the base pressure better than $3 \times 10^{-8} \mathrm{~Pa}$. $E_{\mathrm{F}}$ of the samples was referenced to that of a Au film which was measured frequently during the experiments.

Molecular structures of the neutral and monoanionic picenes were optimized by using the hybrid Hartree-Fock/ density-functional-theory method of Becke ${ }^{12}$ and Lee, Yang, and Parr, ${ }^{13}$ and the $6-31 \mathrm{G}^{*}$ basis set. ${ }^{14}$ The GAUSSIAN 03 program package ${ }^{15}$ was used for our theoretical analyses. This level of theory is, in our experience, sufficient for reasonable descriptions of the geometric and vibrational features of hydrocarbons.

\section{RESULTS AND DISCUSSION}

Figure 1 shows valence band spectra of solid pristine picene measured using incident photon energies of 1100 and $100 \mathrm{eV}$, together with a calculated valence band of pristine picene. The experimental spectrum of pristine picene measured at $1100 \mathrm{eV}$ consists of mainly three regions (I, II, and III): a broad and higher intensity structure from 16 to $23 \mathrm{eV}$, a peak at $14.5 \mathrm{eV}$, and a lower intensity region with several structures between the Fermi level $\left(E_{\mathrm{F}}\right)$ and $13 \mathrm{eV}$ binding energy. The intensity close to $E_{\mathrm{F}}$ is negligible, consistent with the semiconducting nature of solid pristine picene. Change of electronic structure regarding semiconductor to metal transition will be discussed in relation to K-doped picene data later. It is known that valence-band structures of carbon systems (graphite, diamond, and hydrocarbons ${ }^{16}$ ) are formed from C $2 s$ and $2 p$ orbitals with the energy position of C $2 p$ closer to $E_{\mathrm{F}}$. Hybridization between these orbitals makes the band width wider with unchanged relative energy, leading to the $\mathrm{C} 2 s$ dominant band at higher binding energy and the $\mathrm{C} 2 p$ dominant band closer to $E_{\mathrm{F}}$, with the $2 s-p$ states at the region where $2 s$ and $2 p$ states overlap. ${ }^{16}$ Thus one may tentatively assign the states at the regions I, II, and III to C $2 s, \mathrm{C} 2 s-p$, and C $2 p$ dominant states, respectively. This assignment can be checked experimentally with photonenergy dependence of valence band spectra. In the valence band spectrum at $100 \mathrm{eV}$ as compared with that at $1100 \mathrm{eV}$,

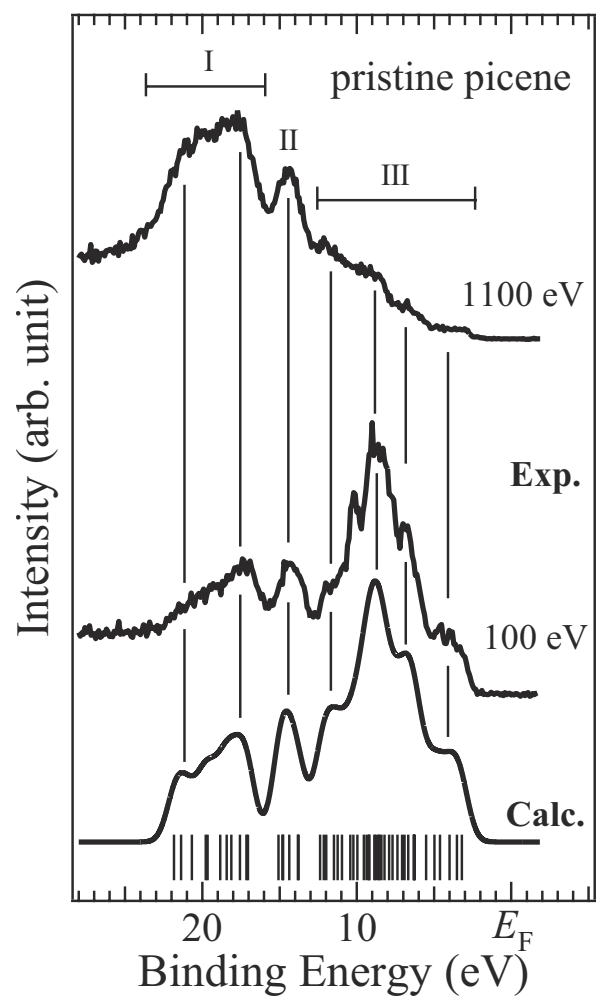

FIG. 1. Incident energy dependence of valence-band spectra from pristine picene using photon energy of 1100 and $100 \mathrm{eV}$, together with molecular-orbital calculation of pristine picene. The calculated spectrum was obtained from calculated molecular-orbital levels convoluted with Gaussian (FWHM of $0.7 \mathrm{eV}$ ) to reproduce the observed spectrum.

the intensity of the region III is strongly enhanced while that of the region I is reduced, if the spectra are normalized to the intensity of the peak at $13 \mathrm{eV}$. As the cross-section ratio of $\mathrm{C} 2 s$ electrons to C $2 p$ electrons is the order of $10^{2}$ for 1100 $\mathrm{eV}$ but is the order of unity for $100 \mathrm{eV},{ }^{11}$ this agrees well with the considerations above, leading to a conclusion that C $2 s$ states dominate the density of states (DOS) from 16 to $23 \mathrm{eV}$ (region I), C $2 p$ states dominate DOS from 2 to $13 \mathrm{eV}$ (region III), and the peak at $14.5 \mathrm{eV}$ consists of C $2 s$ states mixed with $\mathrm{C} 2 p$ states (region II).

As for the region III, one sees several fine structures. We compare the valence band spectra of pristine picene with the molecular-orbital calculation of pristine picene. The vertical bars at bottom are the calculated energy levels. The symmetries of the LUMO and the highest occupied molecular orbitals (HOMO) are $a_{2}$ and $b_{1}$, respectively. The calculated spectrum is obtained from calculated molecular-orbital levels convoluted with Gaussian [full width at half maximum (FWHM) of $0.7 \mathrm{eV}]$ to reproduce the observed spectrum. The use of a larger FWHM of Gaussian than the energy resolution suggests that energy levels of molecular orbitals are dispersed by crystallizing. The cross sections of $\mathrm{C} 2 p$ and $2 s$ are not taken into consideration, assuming that they are comparable at photon energy of $100 \mathrm{eV}$. As indicated with the vertical solid lines, the observed peak structures can be corresponded to calculated structures very well, except for the sharp peak at $11 \mathrm{eV}^{17}$ This result, as is expected for the 


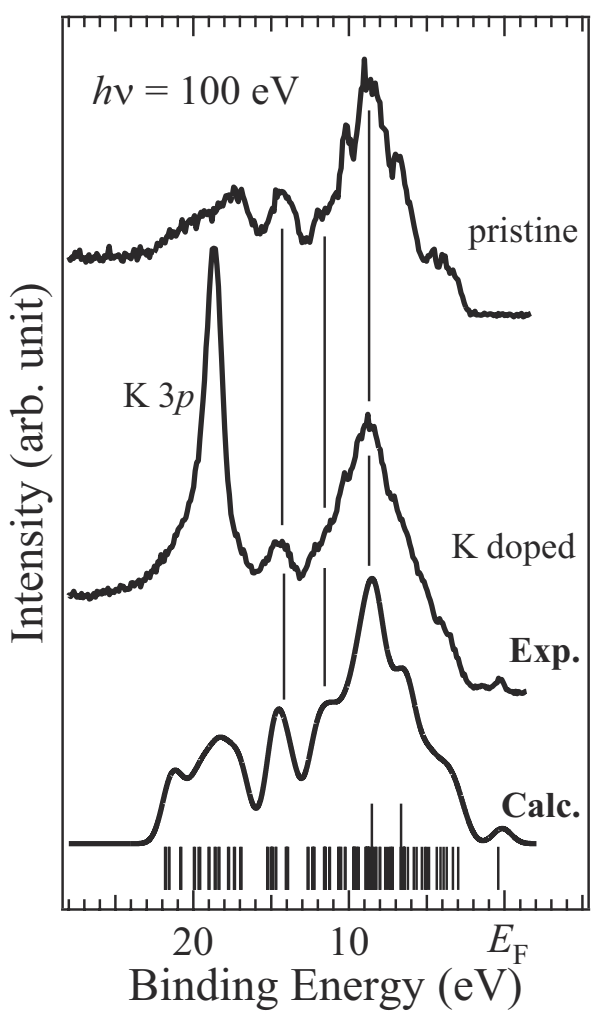

FIG. 2. Valence-band spectrum from K-doped picene using 100 $\mathrm{eV}$ photon energy, together with molecular-orbital calculation of monoanion picene. The calculated spectrum was obtained from calculated molecular-orbital levels for monoanion picene convoluted with Gaussian (FWHM of $0.7 \mathrm{eV}$ ) to reproduce the observed spectrum.

molecular crystal, indicates that the valence band of solid pristine picene dominantly reflects its molecular electronic structure.

In Fig. 2, we show the valence band spectrum of K-doped picene measured with photon energy of $100 \mathrm{eV}$. Using incident energy of $100 \mathrm{eV}$ was crucial to observe electronic structure change near $E_{\mathrm{F}}$, as the spectral change near $E_{\mathrm{F}}$ was not observed clearly for the spectrum with $1100 \mathrm{eV}$ due to the lower photoionized cross sections. ${ }^{18}$ The highest intensity peak at $19 \mathrm{eV}$, which overlaps with the $\mathrm{C} 2 s$ dominant bands, is a $\mathrm{K} 3 p$ shallow core level. The spectral shape with a prominent peak around $8 \mathrm{eV}$ and the peak at $14.5 \mathrm{eV}$ is similar to that of pristine picene, though the shape of $\mathrm{K}$-doped picene has less fine structure compared to that of pristine picene. Regarding energy positions, we found that the 8 and $14.5 \mathrm{eV}$ peaks in the K-doped spectrum are larger by $0.5 \mathrm{eV}$ than those in pristine picene. On the other hand, one can see some differences between the two spectra especially in a lower binding energy region $\left(E_{\mathrm{F}}-6 \mathrm{eV}\right)$. As is evident, the steep edge structure around $3.0 \mathrm{eV}$ seen in pristine picene, which corresponds to states derived from the HOMO band, is not clearly observed in the K-doped picene spectrum. This suggests transformation of HOMO band due to $\mathrm{K}$ doping. Indeed, we found that the valence band spectrum of $\mathrm{K}$-doped picene, especially for the HOMO derived states $\left(E_{\mathrm{B}} \approx 3 \mathrm{eV}\right)$, is not explained by the calculated spectrum of pristine picene very well. More importantly in the

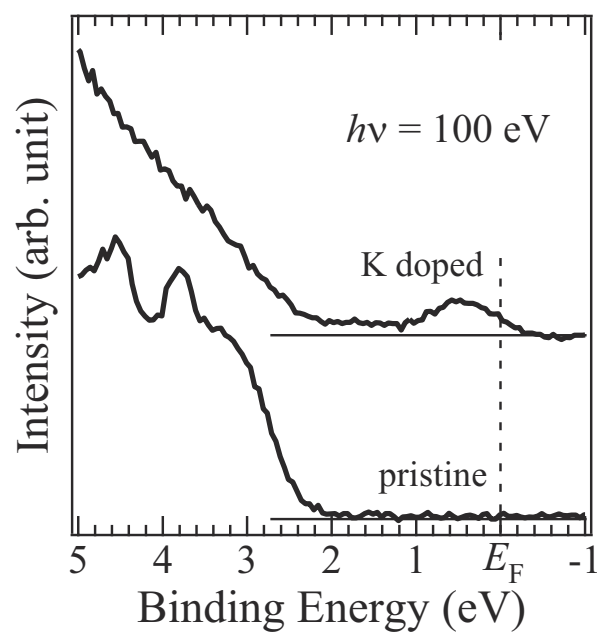

FIG. 3. Valence-band spectra near $E_{\mathrm{F}}$ from pristine picene and $\mathrm{K}$-doped picene using $100 \mathrm{eV}$ photon energy. The horizontal line of each spectrum denotes a background level.

vicinity of $E_{\mathrm{F}}$, a new structure appears in K-doped picene, for which no peak corresponding to this new structure is seen in the spectrum of pristine picene.

Change in the vicinity of $E_{\mathrm{F}}$ by $\mathrm{K}$ doping can be seen in Fig. 3 in more detail. In pristine picene, a steep spectral cutoff is located at $2.7 \mathrm{eV}$, corresponds to the HOMO band, and the tail extends to $2.0 \mathrm{eV}$ binding energy, which is referred as the valence band maximum, as shown as a deviation from the zero line in Fig. 3. This binding energy of the valence band maximum is nearly half of the band gap, ${ }^{7}$ suggesting that solid pristine picene is an intrinsic semiconductor. We also observed no intensity at $E_{\mathrm{F}}$, consistent with semiconducting properties of pristine picene. In K-doped picene, on the other hand, there is a new peak near $E_{\mathrm{F}}$. This result agrees well with the metallic properties of $\mathrm{K}$-doped picene. Note that the new peak near $E_{\mathrm{F}}$ is not a PES signal from $\mathrm{K}$ metal resides on the surface region but one intrinsic to $\mathrm{K}$-doped picene, as the spectral shape of the $\mathrm{K} 3 p$ shallow core level (Fig. 2) does not have plasmon features characteristic of $\mathrm{K} 3 p$ shape of $\mathrm{K}$ metal. The new peak has a maximum around $0.6 \mathrm{eV}$ and a clear Fermi edge could not be observed within our experimental accuracy. The spectral shape of the new states observed in $\mathrm{K}$-doped picene is reminiscent of the spectra of $\mathrm{K}_{3} \mathrm{C}_{60}$ measured at room temperature. The PES spectra of $\mathrm{K}_{3} \mathrm{C}_{60}$ show a broad structure around $0.5 \mathrm{eV}$, which has been attributed to correlationinduced states. ${ }^{9,19-22}$ High-resolution PES studies of $\mathrm{K}_{3} \mathrm{C}_{60}$ at lower temperature revealed presence of a metallic edge with fine structures that have been explained with electronphonon and electron-plasmon interactions. ${ }^{20,21}$ Highresolution PES studies at lower temperature of $\mathrm{K}$-doped picene will provide insight into the superconductivity, as in K-doped fullerides.

Since the energy separation between the bottom edge of the new band near $E_{\mathrm{F}}$ and the valence-band maximum of $\mathrm{K}$-doped picene is about $1.0 \mathrm{eV}$ and smaller than the binding energy of the valence-band maximum $(2.0 \mathrm{eV})$ of pristine picene, the new peak of K-doped picene cannot be explained by simple rigid LUMO band shift of pristine picene due to 
the filling with electrons. Possibility of doping induced localized states, like in an iodine-doped pentacene, ${ }^{23}$ as the origin of the new states may be ruled out, since a simple photoionization cross-section analysis showed that the new states have negligible $\mathrm{K} 4 s$ character. The cross section of $\mathrm{K} 4 s$ has $10^{-2}$ times smaller than that of $\mathrm{K} 3 p,{ }^{11}$ which would make the intensity of the new states at $E_{\mathrm{F}}$ negligible in the spectrum shown in Fig. 2. In view of the transformation of the HOMO band by $\mathrm{K}$ doping, the new peak may be considered as the states derived from the LUMO band of pristine picene changed by $\mathrm{K}$ doping. These indicate that HOMO and LUMO bands are modified by $\mathrm{K}$ doping, and the electronic structure of $\mathrm{K}$-doped picene is, thus, not obtained by a simple rigid-band shift of that of pristine picene. Nonrigid bandlike change in band dispersion was predicted from recent first-principles calculations, ${ }^{24}$ where doped-K atoms and more importantly change in the molecular orientation influence the electronic band structure derived from LUMO and LUMO +1 of pristine picene. However, band-structure calculations for K-doped picene based on experimental crystal structure data in order to be compared with the present PES spectrum have not been available so far.

For alkali-doped fullerene, importance of electronintramolecular-vibration interaction has been proposed for observed nonrigid band change, as mentioned above. In order to study the electron-intermolecular vibration interaction in K-doped picene, we discuss the electronic structure of $\mathrm{K}$-doped picene by comparing the overall valence band structure with molecular-orbital calculations taking electronintramolecular-vibration interactions into consideration (Fig. 2 ). Here we use a calculated result where one electron is doped into a picene molecule (monoanion picene: $\mathrm{C}_{22} \mathrm{H}_{14}^{-}$), as estimated from $\mathrm{K}$ concentration $x$ of $1.0 \pm 0.3$. Molecular calculation results of dianion and trianions (not shown) exhibit over all spectral shape similar to monoanion but the intensity of the states near $E_{\mathrm{F}}$ depends on the number of electrons. The symmetries of the state near $E_{\mathrm{F}}$ and the next energy level $\left(E_{\mathrm{B}} \approx 3 \mathrm{eV}\right)$ are $a_{2}$ and $b_{1}$, respectively, which are the same as the LUMO and HOMO of pristine picene. Electron doping causes changes in the HOMO and LUMO levels in picene, coming from strain of a picene molecule due to a coupling between doped electrons in the LUMO of pristine picene and intramolecular phonon of picene molecule. Thus, the calculated spectrum of electron-doped picene is different from that of pristine picene, evidently at the structure around $3 \mathrm{eV}$. The observed spectral shape with peaks at 8 and $14.5 \mathrm{eV}$ reveals an almost monotonous decrease of intensity around $2-8 \mathrm{eV}$, and the structure near $E_{\mathrm{F}}$ are well reproduced with that of the molecular-orbital calcu- lation for electron-doped picene as indicated with the vertical solid lines. Excellent agreement between experiments and calculations indicates that electron-intramolecular-vibration coupling between electrons doped into the LUMO and intramolecular phonon of picene molecule makes the electronic structure of K-doped picene different from that of pristine picene. According to the calculations, the electronphonon coupling constants of picene become stronger with an increase in the number of electrons per picene molecule. This is in line with the observation that $\mathrm{K}$-doped picene $\left(\mathrm{K}_{x} \mathrm{C}_{22} \mathrm{H}_{14}\right)$ is found to become a superconductor for $x \approx 3$, corresponding to three electrons doping into picene. These results suggest that the electron-intramolecular-vibration coupling is crucial to understand the physical properties including superconductivity in K-doped picene, ${ }^{25}$ as discussed for fullerides ${ }^{10}$ and bisethylenedithio-teterahiafuvalene (BEDT-TTF). ${ }^{26}$

\section{CONCLUSIONS}

We have performed PES studies of solid pristine and $\mathrm{K}$-doped picene in order to study the electronic structure of solid picene and its evolution with $\mathrm{K}$ doping. The valence band spectrum of pristine solid picene consists of mainly three structures that can be ascribed to $\mathrm{C} 2 s, 2 s-p$, and $2 p$ dominant states, and no states at $E_{\mathrm{F}}$, consistent with semiconducting properties. The spectral shape is well explained with broadened molecular orbital calculations, indicating the molecular nature of solid picene. The valence band spectrum of K-doped picene shows an overall valence band spectral shape similar to that of pristine solid picene with a $\mathrm{K} 3 p$ shallow core level. However, larger differences are observed for the HOMO derived bands and states near $E_{\mathrm{F}}$, latter of which is consistent with metallic properties. The change in the valence band due to $\mathrm{K}$ doping cannot be explained in terms of a simple band-filling picture with doped electrons. Comparison with available theoretical results shows that the new peak near $E_{\mathrm{F}}$ corresponds to states transformed from LUMO of pristine solid picene due to electronintramolecular-vibration coupling caused by electron doping. This result points to importance of electron-intramolecularvibration coupling of the K-doped picene.

\section{ACKNOWLEDGMENTS}

This work was supported partly by a Grant-in-Aid for Scientific Research of the Ministry of Education, Culture, Sports, Science and Technology, Japan (Grant No. 20340091). The measurement at BL27SU of SPring-8 was performed under Proposal No. 2009B1697.
${ }^{1}$ E. A. Ekimov, V. A. Sidorov, E. D. Bauer, N. N. Mel'nik, N. J. Curro, J. D. Thompson, and S. M. Stishov, Nature (London) 428, 542 (2004).

${ }^{2}$ Y. Takano, T. Takenouchi, S. Ishii, S. Ueda, T. Okutsu, I. Sakaguchi, H. Umezawa, H. Kawarada, and M. Tachiki, Diamond
Relat. Mater. 16, 911 (2007).

${ }^{3}$ T. E. Weller, M. Ellerby, S. S. Saxena, R. P. Smith, and N. T. Skipper, Nat. Phys. 1, 39 (2005); N. Emery, C. Hérold, M. d'Astuto, V. Garcia, Ch. Bellin, J. F. Marêché, P. Lagrange, and G. Loupias, Phys. Rev. Lett. 95, 087003 (2005). 
${ }^{4}$ A. Y. Ganin, Y. Takabayashi, Y. Z. Khimyak, S. Margadonna, A. Tamai, M. J. Rosseinsky, and K. Prassides, Nature Mater. 7, 367 (2008).

${ }^{5}$ R. Mitsuhashi, Y. Suzuki, Y. Yamanari, H. Mitamura, T. Kambe, N. Ikeda, H. Okamoto, A. Fujiwara, M. Yamaji, N. Kawasaki, Y. Maniwa, and Y. Kubozono, Nature (London) 464, 76 (2010).

${ }^{6}$ A. De, R. Ghosh, S. Roychowdhury, and P. Roychowdhury, Acta Crystallogr., Sect. C: Cryst. Struct. Commun. 41, 907 (1985).

${ }^{7}$ H. Okamoto, N. Kawasaki, Y. Kaji, Y. Kubozono, A. Fujiwara, and M. Yamaji, J. Am. Chem. Soc. 130, 10470 (2008).

${ }^{8}$ N. Kawasaki, Y. Kubozono, H. Okamoto, A. Fujiwara, and M. Yamaji, Appl. Phys. Lett. 94, 043310 (2009).

${ }^{9}$ T. Takahashi, S. Suzuki, T. Morikawa, H. Katayama-Yoshida, S. Hasegawa, H. Inokuchi, K. Seki, K. Kikuchi, S. Suzuki, K. Ikemoto, and Y. Achiba, Phys. Rev. Lett. 68, 1232 (1992).

${ }^{10}$ O. Gunnarsson, Rev. Mod. Phys. 69, 575 (1997).

${ }^{11}$ J. J. Yeh and I. Lindau, At. Data Nucl. Data Tables 32, 1 (1985).

${ }^{12}$ A. D. Becke, Phys. Rev. A 38, 3098 (1988); J. Chem. Phys. 98, 5648 (1993).

${ }^{13}$ C. Lee, W. Yang, and R. G. Parr, Phys. Rev. B 37, 785 (1988).

${ }^{14}$ R. Ditchfield, W. J. Hehre, and J. A. Pople, J. Chem. Phys. 54, 724 (1971); P. C. Hariharan and J. A. Pople, Theor. Chim. Acta 28, 213 (1973).

${ }^{15}$ M. J. Frisch et al., GAUSSIAN 03, Gaussian, Inc.: Wallingford CT, 2004.

${ }^{16}$ J. J. Pireaux, S. Svensson, E. Basilier, P-Á Malmqvist, U. Gelius, R. Caudano, and K. Siegbahn, Phys. Rev. A 14, 2133 (1976).

${ }^{17}$ This structure can be corresponded to a shoulder structure seen in calculated spectra broadened with Gaussian with a narrower
FWHM (not shown). Observable deviation may be related the fact that the orbitals of this region have an in-plane sigmalike character and may have larger influence from the induced photoholes. This may be in line with the reduction in this structure in K-doped picene.

${ }^{18} \mathrm{PES}$ measurements using $100 \mathrm{eV}$ photon may dominantly reflect a surface-derived electronic structure that may be different from bulk electronic structure. As shown in text, however, the observed spectral change due to $\mathrm{K}$ doping is consistent with physical properties of K-doped picene, suggesting that what we observe reflects the bulk electronic structure of K-doped picene.

${ }^{19}$ P. J. Benning, F. Stepniak, and J. H. Weaver, Phys. Rev. B 48, 9086 (1993).

${ }^{20}$ M. Knupfer, M. Merkel, M. S. Golden, J. Fink, O. Gunnarsson, and V. P. Antropov, Phys. Rev. B 47, 13944 (1993).

${ }^{21}$ A. Goldoni, S. L. Friedmann, Z.-X. Shen, and F. Parmigiani, Phys. Rev. B 58, 11023 (1998).

${ }^{22}$ T. Käämbre, J. Schiessling, L. Kjeldgaard, L. Qian, I. Marenne, J. N. O'Shea, J. Schnadt, D. Nordlund, C. J. Glover, J.-E. Rubensson, P. Rudolf, N. Mårtensson, J. Nordgren, and P. A. Brühwiler, Phys. Rev. B 75, 195432 (2007).

${ }^{23}$ Y. Shichibu and K. Watanabe, Jpn. J. Appl. Phys., Part 1 42, 5472 (2003).

${ }^{24}$ T. Kosugi, T. Miyake, S. Ishibashi, R. Arita, and H. Aoki, J. Phys. Soc. Jpn. 78, 113704 (2009).

${ }^{25}$ T. Kato and T. Yamabe, J. Phys. Chem. A 109, 4804 (2005).

${ }^{26}$ E. Demiralp, S. Dasgupta, and W. A. Goddard III, J. Am. Chem. Soc. 117, 8154 (1995). 